PROCEEDINGS OF THE

AMERICAN MATHEMATICAL SOCIETY

Volume 134, Number 7 , Pages 2067-2078

S 0002-9939(06)08214-

Article electronically published on January 6, 2006

\title{
KMS STATES ON C*-ALGEBRAS ASSOCIATED TO EXPANSIVE MAPS
}

\author{
ALEX KUMJIAN AND JEAN RENAULT
}

(Communicated by David R. Larson)

\begin{abstract}
Using Walters' version of the Ruelle-Perron-Frobenius Theorem we show the existence and uniqueness of KMS states for a certain one-parameter group of automorphisms on a $\mathrm{C}^{*}$-algebra associated to a positively expansive map on a compact metric space.
\end{abstract}

\section{INTRODUCTION}

Operator algebras have long been used to model the algebra of observables in quantum theory, and time evolution is often given by a one-parameter group of automorphisms of this algebra. A state of the system is given by a state of the operator algebra. In the $\mathrm{C}^{*}$-algebraic formulation of quantum statistical mechanics, KMS states are equilibrium states associated to the one-parameter group of automorphisms of time evolution (see $\underline{\mathrm{BR}}, \S 5.3]$ ). The question of uniqueness of KMS state is therefore of obvious physical interest and has thus been an object of much study by operator algebraists. Olesen and Pedersen showed that the gauge action on the Cuntz algebra $\mathcal{O}_{n}$ has a unique KMS state, and this occurs at inverse temperature $\log n$ (see [OP]). The Cuntz algebra $\mathcal{O}_{n}$ may profitably be viewed as arising from a groupoid associated to the one-sided Bernoulli shift on $n$ letters, which not coincidentally has topological entropy of $\log n$. The one-sided Bernoulli shift is a standard example of a positively expansive local homeomorphism studied in topological dynamics (see [W2]). We generalize the work of Olesen and Pedersen using results from topological dynamics and groupoid techniques (see Remark 3.6).

Our main result concerns the $\mathrm{C}^{*}$-algebra associated to a positively expansive exact local homeomorphism $T$ defined on a compact metric space $X$ (see [D, R3]). A continuous real-valued function $\varphi$ on $X$ gives rise to a one-parameter group of automorphisms on the $\mathrm{C}^{*}$-algebra. We apply Walters' version of the Ruelle-PerronFrobenius Theorem and groupoid techniques to establish existence and uniqueness of KMS states under mild hypotheses on $\varphi$. In particular, if $\varphi$ is positive and Hölder continuous there is a unique KMS state (this generalizes recent work of Exel; cf. Ex3).

When a $\mathrm{C}^{*}$-algebra $A$ is written as the $\mathrm{C}^{*}$-algebra of a groupoid $G$ (by the construction of [R1]), a natural problem is to describe its structure in terms of $G$. For example, positive results about the ideal structure (see [R2]) or the K-theory

Received by the editors November 11, 2003 and, in revised form, February 15, 2005.

2000 Mathematics Subject Classification. Primary 46L05, 46L30, 46L40.

(C)2006 American Mathematical Society Reverts to public domain 28 years from publication 
(see $\left[\mathrm{T}\right.$ ) of groupoid $\mathrm{C}^{*}$-algebras have been obtained, especially in the amenable case. The scope of this paper is more limited. Let $G$ be a locally compact groupoid with Haar system (for convenience, we assume that $G$ is étale and Hausdorff) and let $C^{*}(G)$ be its $\mathrm{C}^{*}$-algebra. Every continuous cocycle $c \in Z^{1}(G, \mathbf{R})$ defines a one-parameter automorphism group $\alpha_{c}$ of $C^{*}(G)$ as in [R1, Section II.5]. First, we show in Proposition 3.2 that if $c^{-1}(0)$ is principal, then the KMS states of $\alpha_{c}$ at inverse temperature $\beta$ are exactly the quasi-invariant probability measures which admit $e^{-\beta c}$ as Radon-Nikodym derivative. This extends [R1, Proposition II.5.4], which gave a complete answer only when $G$ itself is principal. There certainly exist improvements of this result (see Remark 3.4), but it suffices for our purpose. Then, we apply this result to the Cuntz-like $\mathrm{C}^{*}$-algebras of [D, R3]. These algebras are of the form $C^{*}(G)$, where $G=G(X, T)$ and $(X, T)$ is a dynamical system where $X$ is a compact space and $T$ is a surjective local homeomorphism (see below). Proposition 3.2 reduces the KMS problem to a Radon-Nikodym problem which fits the framework of the thermodynamical formalism. Under classical assumptions on the dynamical system $(X, T)$ and on the potential $\varphi \in C(X, \mathbf{R})$ defining the cocycle $c_{\varphi} \in Z^{1}(G, \mathbf{R})$, Ruelle's Perron-Frobenius theorem can be applied to obtain existence and uniqueness results. Theorem 3.5 summarizes our results about KMS states of these automorphism groups. We assume that $T$ is positively expansive and exact (see Proposition 2.1). We first give a necessary and sufficient condition for the existence of KMS states at inverse temperature $\beta$, namely the equation $P(T,-\beta \varphi)=0$, where $P$ is the pressure. Under the assumption that $\varphi$ satisfies

the Walters condition, we give a necessary condition $\left(c_{\varphi}^{-1}(0)\right.$ is principal) for the existence of KMS states. If $\varphi$ satisfies the Bowen condition and has constant sign, then we have the existence and the uniqueness of the KMS inverse temperature and state. Our results, which complete [R4, Section 4.2], strictly cover previous results about KMS states of gauge one-parameter automorphism groups of Cuntz and Cuntz-Krieger algebras obtained by a number of authors (e.g. OP, Ev, EFW, [Z, [KP, Ex2]). Although they do not use groupoid techniques as we do, Kerr-Pinzari in $\mathrm{KP}$ and Exel in Ex2 also make essential use of Ruelle's transfer operator.

Our work is organized as follows. In Section 2, we recall the definition of the groupoid $G(X, T)$ and some facts pertaining to the dynamics of a positively expansive map on a compact metrice space. Section 3 contains our results about KMS states and a list of examples. Besides the cases previously studied, we give an example of a unique KMS state for a potential which does not have a constant sign. Section 4 gives some properties of the associated crossed product $\mathrm{C}^{*}$-algebras.

The first author thanks the Université d'Orléans for its support.

\section{Preliminaries}

For $X$ a locally compact space and $T: X \rightarrow X$ a local homeomorphism, we let $G=G(X, T)$ denote the associated groupoid (see [D, R3]), where

$$
G(X, T)=\left\{(x, m-n, y): T^{m} x=T^{n} y\right\} .
$$

This is an étale groupoid (a topological groupoid with structure maps that are local homeomorphisms); note that the unit space $G^{0}$ may be identified with $X$ via the map $x \mapsto(x, 0, x)$. The subgroupoid for which $m=n$, denoted $R=R(X, T)$, is an approximately proper equivalence relation (see [R4, R5]). Note that $R=\bigcup_{n} R_{n}$, 
where $(x, y) \in R_{n}$ if $T^{n} x=T^{n} y$ and $C^{*}\left(R_{n}\right)$ is strongly Morita equivalent to $C_{0}(X)$.

Let $X$ be a compact metric space with metric $\rho$ which is fixed throughout and assumed to contain infinitely many points. A continuous surjection $T: X \rightarrow X$ is said to be positively expansive if there is an $\epsilon>0$ such that if $x \neq y$, then $\rho\left(T^{n} x, T^{n} y\right) \geq \epsilon$ for some $n \geq 0$. Note that this property does not depend on the choice of the metric and can be expressed topologically as follows: there is an open subset $U$ of $X \times X$ which contains the diagonal such that for all $x \neq y$ there is an $n \in \mathbf{N}$ with $\left(T^{n} x, T^{n} y\right) \notin U$. Observe that $T$ is not a homeomorphism, for we have assumed that $X$ is not finite (see [AH, 2.2.12]).

Let $T: X \rightarrow X$ be positively expansive. By $\mathrm{Rd}$ there are an equivalent metric $\rho^{\prime}$ and constants $\tau>0, \kappa>1$ such that if $\rho^{\prime}(x, y)<\tau$, then $\rho(T x, T y) \geq \kappa \rho^{\prime}(x, y)$. If $T$ is open, then it is a local homeomorphism (such a map is called expanding in $[\mathrm{AH}]$ ); see [AH, 2.2.21] for an example due to Rosenholtz of a positively expansive map which is not open and therefore not a local homeomorphism.

Let $T: X \rightarrow X$ be a local homeomorphism. Then $T$ is said to be exact if for every non-empty open set $U \subset X$ there is an $n>0$ such that $T^{n}(U)=X$ (this property is called mixing in [FJ, §2]). We say that $T$ satisfies the weak specification condition if for every $\epsilon>0$ there is $n>0$ such that for all $x \in X, T^{-n} x$ is $\epsilon$-dense, that is, we have

$$
X=\bigcup_{y \in T^{-n} x} B(y, \epsilon),
$$

where $T^{-n} x=\left\{y \in X \mid T^{n} y=x\right\}$ and $B(y, \epsilon)$ is the open ball of radius $\epsilon$ centered at $y$ (cf. [W3, 1.2], where this is listed as one of four equivalent conditions for a positively expansive local homeomorphism).

Proposition 2.1. Let $T: X \rightarrow X$ be a local homeomorphism. Then the following conditions are equivalent:

(i) $R$ is minimal.

(ii) $T$ is exact.

(iii) $T$ satisfies the weak specification condition.

Proof. The equivalence of (i) and (ii) follows by the compactness of $X$. For the minimality of $R$ is equivalent to the condition that the saturation of any non-empty open set is all of $X$. Now suppose that (ii) holds and fix $\epsilon>0$. By compactness there are $x_{1}, \ldots, x_{m} \in X$ such that $X \subset \bigcup_{i} B\left(x_{i}, \epsilon / 2\right)$. By (ii) there is $n>0$ such that $T^{n}\left(B\left(x_{i}, \epsilon / 2\right)\right)=X$ for $i=1, \ldots, m$. To show that $T$ satisfies the weak specification condition, we verify that $T^{-n} x$ is $\epsilon$-dense for every $x \in X$. Given $x, z \in X$ there is an $i$ such that $\rho\left(z, x_{i}\right)<\epsilon / 2$. Since $T^{n}\left(B\left(x_{i}, \epsilon / 2\right)\right)=X, B\left(x_{i}, \epsilon / 2\right) \cap T^{-n} x \neq \emptyset$. Choose an element $y$ in this set; then we have

$$
\rho(z, y) \leq \rho\left(z, x_{i}\right)+\rho\left(x_{i}, y\right)<\epsilon,
$$

and $T^{-n} x$ is $\epsilon$-dense as required. Now suppose that $T$ satisfies the weak specification condition. We verify that $R$ is minimal, that is, every orbit under $R$ is dense. Given $x \in X$, the orbit of $x$ is given by $\bigcup_{n} T^{-n}\left(T^{n} x\right)$; for $\epsilon>0$, there is $n>0$ so that $T^{-n}\left(T^{n} x\right)$ is $\epsilon$-dense by weak specification. Hence, the orbit of $x$ is dense.

Example 2.2. Fix $n \in \mathbf{N}, n>1$, and let $X=\left\{\left(x_{0}, x_{1}, \ldots\right) \mid x_{i}=1,2, \ldots, n\right\}$. For $x, y \in X$ with $x \neq y$, set $\rho(x, y)=2^{-j}$ where $j=\min \left\{i \mid x_{i} \neq y_{i}\right\}$ and define $T: X \rightarrow X$ by $T\left(x_{0}, x_{1}, \ldots\right)=\left(x_{1}, x_{2}, \ldots\right)$. Then $X$ is a compact metric 
space and $T$ is a positively expansive local homeomorphism which satisfies the weak specification condition. The map $T$ is often called the Bernoulli shift on $n$ letters. Note that $C^{*}(G)$ is the Cuntz algebra $\mathcal{O}_{n}$ (see $[\mathrm{Cu}$ ), which is generated by $n$ isometries $S_{1}, \ldots, S_{n}$ subject to the relation

$$
1=\sum_{j=1}^{n} S_{j} S_{j}^{*}
$$

Example 2.3 (see [CK]). Let $A$ be an irreducible $n \times n$ zero-one matrix $A=$ $(A(i, j))_{1 \leq i, j \leq n}$. Then $A$ defines a closed subset $X_{A}$ of $X$ above

$$
X_{A}=\left\{x \in X: A\left(x_{i}, x_{i+1}\right)=1\right\} .
$$

Note that $T X_{A} \subset X_{A}$ and let $T_{A}: X_{A} \rightarrow X_{A}$ denote the restriction. Such a map is called a subshift of finite type. Note that $T_{A}$ is a positively expansive local homeomorphism, but it does not necessarily satisfy the weak specification condition unless $A$ is primitive (i.e. there is a positive integer $k$ such that all entries of $A^{k}$ are nonzero). Note that $C^{*}(G) \cong \mathcal{O}_{A}$, where $\mathcal{O}_{A}$ is the Cuntz-Krieger algebra associated to the matrix $A ; \mathcal{O}_{A}$ is also generated by $n$ isometries.

Example 2.4. Fix $n>1$; let $X=\mathbf{T}=\mathbf{R} / \mathbf{Z}$ and define $T: X \rightarrow X$ by $T x=n x$. We endow $X$ with the arclength metric. Then $T$ is a positively expansive local homeomorphism which satisfies the weak specification condition. More generally an integer $k \times k$ matrix, all of whose eigenvalues exceed one in absolute value, defines a positively expansive local homeomorphism on $X=\mathbf{T}^{k}=\mathbf{R}^{k} / \mathbf{Z}^{k}$ (see $[\mathrm{AH}]$ ).

Let $C(X ; \mathbf{R})$ denote the space of continuous real-valued functions on $\mathbf{R}$. Then for $\varphi \in C(X ; \mathbf{R})$ we define the transfer operator $\mathcal{L}_{\varphi}: C(X ; \mathbf{R}) \rightarrow C(X ; \mathbf{R})$ by the formula

$$
\left(\mathcal{L}_{\varphi} f\right)(x)=\sum_{y \in T^{-1} x} e^{\varphi(y)} f(y) .
$$

The following proposition is an easy consequence of Theorem 1.3 and Corollary 2.3 of [W3]. The pressure $P(T, \varphi)$ of $T$ at $\varphi$ is defined in [W2, §9.1].

Proposition 2.5. Let $T: X \rightarrow X$ be a local homeomorphism which is positively expansive and exact, and let $\varphi \in C(X ; \mathbf{R})$. There is a unique $\lambda>0$ such that

$$
\mathcal{L}_{\varphi}^{*} \mu=\lambda \mu
$$

for some probability measure $\mu$ on $X$. Moreover, $\log \lambda=P(T, \varphi)$, and the sequence $\frac{1}{n} \log \left(\mathcal{L}_{\varphi}^{n} 1\right)$ converges uniformly to the constant $P(T, \varphi)$.

Note that $P(T, 0)=h(T)$, the topological entropy of $T$ (see [W2, 9.7i]).

Corollary 2.6. Let $T: X \rightarrow X$ be as above. Then $h(T)>0$.

Proof. Apply the above proposition with $\varphi=0$. Then there is a probability measure $\mu$ on $X$ such that

$$
\mathcal{L}_{0}^{*} \mu=e^{h(T)} \mu .
$$

Arguing as in [R3, Corollary 4.3] we may deduce that $\mu$ is quasi-invariant under $G$. By Proposition 2.1, $R$ is minimal, and therefore $\mu$ must be faithful. We have $\mathcal{L}_{0}(1)>1$. Hence

$$
e^{h(T)}=\mathcal{L}_{0}^{*} \mu(1)=\mu\left(\mathcal{L}_{0}(1)\right)>\mu(1)=1 .
$$

Thus $h(T)>0$ as required. 
To ensure that the measure $\mu$ is unique we will need to impose a condition on the function $\varphi$.

Definition 2.7 (see [W3, §1], $[\mathrm{Ru}]$ ). A function $\varphi \in C(X ; \mathbf{R})$ is said to satisfy the Bowen condition with respect to $T: X \rightarrow X$ if there are $\delta, C>0$ such that

$$
\sum_{i=0}^{n-1} \varphi\left(T^{i} x\right)-\varphi\left(T^{i} y\right) \leq C
$$

for all $x, y \in X$ and $n>0$ such that $d\left(T^{i} x, T^{i} y\right) \leq \delta$ for $0 \leq i \leq n-1$.

The Walters condition introduced in [W1 by $\mathrm{P}$. Walters requires that $C$ can be chosen arbitrarily small (then $\delta$ depends on $C$ ). Recall that a real-valued function $\varphi$ defined on a compact metric space $(X, \rho)$ is said to be Hölder continuous if there are constants $r, K>0$ such that $|\varphi(x)-\varphi(y)| \leq K \rho(x, y)^{r}$ for all $x, y \in X$. Observe that a Hölder continuous function satisfies the Walters condition. To show this we may assume that we are dealing with a Reddy metric. If $\delta \leq \tau$, then the above sum is not greater than $C=K \delta^{r} \kappa^{r} /\left(\kappa^{r}-1\right)$. If $X$ is a smooth manifold, then differentiability implies Hölder continuity.

By Walters' version of the Ruelle-Perron-Frobenius Theorem (cf. WW3, Theorem 2.16] and [W1, Theorem 8]) we have:

Theorem 2.8. Let $T: X \rightarrow X$ be a local homeomorphism which is positively expansive and exact, and let $\varphi \in C(X ; \mathbf{R})$.

(i) Assume that $\varphi$ satisfies the Bowen condition. Then there are unique $\lambda>0$ and $\mu \in M(X)$ such that

$$
\mathcal{L}_{\varphi}^{*} \mu=\lambda \mu .
$$

(ii) Assume that $\varphi$ satisfies the Walters condition. Then there is also $h \in$ $C(X, \mathbf{R}), h>0$, such that

$$
\mathcal{L}_{\varphi} h=\lambda h .
$$

A proof of the assertion (i) of this theorem is given in R5, Theorem 6.1 and Proposition 7.2].

\section{KMS STATES}

Our main result on existence and uniqueness of KMS states is contained in Theorem 3.5 below. If $G$ is an étale groupoid we let $C^{*}(G)$ denote the full $\mathrm{C}^{*}$-algebra of $G$ as defined in [R1, Chapter II]. Let $T: X \rightarrow X$ be a local homeomorphism which is positively expansive and exact. Then if $G=G(X, T)$ is the groupoid constructed above, $C^{*}(G)$ is purely infinite and simple (see $\mathrm{An}$ ). We shall mainly be concerned with $\mathrm{KMS}$ states on such $\mathrm{C}^{*}$-algebras.

Definition 3.1. Let $A$ be a $\mathrm{C}^{*}$-algebra, let $\beta \in \mathbf{R}$ and let $\alpha: \mathbf{R} \rightarrow \operatorname{Aut}(A)$ be a strongly continuous action. For $\beta \neq 0$, a state $\omega$ on $A$ is said to satisfy the KMS condition for $\alpha$ at inverse temperature $\beta$ if

$$
\omega\left(b \alpha_{i \beta}(a)\right)=\omega(a b)
$$

for all $a, b \in A$ with $a$ entire for $\alpha$. We say that a state $\omega$ satisfies the KMS condition for $\alpha$ at $\beta=0$ if $\omega$ is an $\alpha$-invariant tracial state (see [P, §8.12], [BEK], [BR, §5.3]). 
Let $G$ be an étale groupoid and let $c \in Z^{1}(G, \mathbf{R})$ be a continuous real-valued one-cocycle. Let $\alpha^{c}: \mathbf{R} \rightarrow \operatorname{Aut}\left(C^{*}(G)\right)$ denote the associated action on $C^{*}(G)$ defined for $f \in C_{c}(G)$ by $\left(\alpha_{t}^{c}(f)\right)(\gamma)=e^{i t c(\gamma)} f(\gamma)$ for $\gamma \in G$ and $t \in \mathbf{R}$. Let $\mu$ be a quasi-invariant probability measure on the unit space $G^{(0)}$ and let $\beta \in \mathbf{R}$. Then $\mu$ is said to satisfy the $(c, \beta)$-KMS condition if the modular function of $\mu$ (i.e. the RadonNikodym derivative $d r^{*} \mu / d s^{*} \mu$ ) is $e^{-\beta c}$ (see [R1, Def. I.3.15]). The associated state on $C^{*}(G)$ defined by $\omega_{\mu}(f)=\int_{G^{(0)}} f(x) d \mu(x)$ for $f \in C_{c}(G)$ satisfies the KMS condition for $\alpha$ at inverse temperature $\beta$. It is shown in [R1, Prop. II.5.4] that if $G$ is principal, then every KMS state arises in this fashion. Since a groupoid associated to a local homeomorphism need not be principal, we need an extension of this result. For the sake of simplicity, we only consider the case of a locally compact groupoid which is étale and Hausdorff.

Proposition 3.2. Let $G$ be a Hausdorff étale groupoid. Let $c \in Z^{1}(G, \mathbf{R})$ be a continuous real-valued one-cocycle and let $\alpha$ denote the associated action. Assume that $c^{-1}(0)$ is principal. Then every KMS state for $\alpha$ at inverse temperature $\beta$ is of the form $\omega_{\mu}$ for some quasi-invariant probability measure $\mu$ on $G^{(0)}$ with RadonNikodym derivative $d r^{*} \mu / d s^{*} \mu=e^{-\beta c}$.

Proof. Our assumption means that $c^{-1}(0) \cap G^{\prime} \subset G^{(0)}$, where $G^{\prime}$ is the isotropy bundle $\{\gamma \in G: r(\gamma)=s(\gamma)\}$. Suppose that $\beta \neq 0$; then replacing $c$ by $\beta c$, we may assume that $\beta=1$. Thus, let $\omega$ be a KMS state for $\alpha$ at inverse temperature 1 . Then the restriction of $\omega$ to $C_{0}\left(G^{(0)}\right)$ defines a probability measure $\mu$ on $G^{(0)}$. It is shown in [R1, II.5.4] that $\mu$ is quasi-invariant with the requisite modular function.

The restriction of $\omega$ to $C_{c}(G)$ is a complex Radon measure and defines a complex measure $\nu$ on $G$ ( $\mu$ is the restriction of $\nu$ to $\left.G^{(0)}\right)$. We show just as in [R1, II.5.4] that the support of $\nu$ is contained in the closed set $G^{\prime}$. By the KMS condition, we have $\omega((h \circ r) f)=\omega(f(h \circ s))$ for all $f \in C_{c}(G), h \in C_{c}(X)$. Suppose that the support of $g \in C_{c}(G)$ does not meet $G^{\prime}$. Then there exist open sets $U_{i}, V_{i} \subset G^{(0)}$, $i \in I$ finite, such that $\left\{G_{U_{i}}^{V_{i}}, i \in I\right\}$ covers the support of $g$ and $U_{i} \cap V_{i}=\emptyset$ for all $i \in I$. Using a partition of unity, we can write $g=\sum_{I} g_{i}$, where the support of $g_{i}$ is contained in $G_{U_{i}}^{V_{i}}$. For all $i \in I$, there exist $h_{i} \in C_{c}\left(G^{(0)}\right)$ such that $\left(h_{i} \circ r\right) g_{i}=g_{i}$ and $g_{i}\left(h_{i} \circ s\right)=0$. This implies $\omega\left(g_{i}\right)=0$ and $\omega(g)=0$. Next we note that $\omega$ is invariant under $\alpha$. This implies that for all $f \in C_{c}(G)$ and all $t \in \mathbf{R}$, we have $\omega\left(e^{i t c} f\right)=\omega(f)$. This implies that for all $t \in \mathbf{R}, e^{i t c} \equiv 1$ on the support of $\nu$. Therefore the support of $\nu$ is contained in $c^{-1}(0)$. Thus under our assumption that $c^{-1}(0) \cap G^{\prime}$ is contained in $G^{(0)}$, the measures $\nu$ and $\mu$ agree. Then $\omega$ and $\omega_{\mu}$ agree on $C_{c}(G)$, hence on $C^{*}(G)$.

Now let $\omega$ be a KMS state for $\alpha$ at inverse temperature $\beta=0$; then $\omega$ is an $\alpha$ invariant tracial state. Then a similar argument may be used to show that $\omega=\omega_{\mu}$ for some invariant probability measure $\mu$ on $G^{(0)}$.

Given a local homeomorphism $T: X \rightarrow X$ and $\varphi \in C(X ; \mathbf{R})$, we construct the groupoid $G=G(X, T)$ as above. Every $\varphi \in C(X ; \mathbf{R})$ defines a continuous one-cocycle $c=c_{\varphi} \in Z^{1}(G, \mathbf{R})$ by the formula

$$
c_{\varphi}(x, m-n, y)=\sum_{i=0}^{m-1} \varphi\left(T^{i} x\right)-\sum_{j=0}^{n-1} \varphi\left(T^{j} y\right) .
$$


Moreover, every element of $Z^{1}(G, \mathbf{R})$ is of this form (see [DKM]). In that case, the condition $c_{\varphi}^{-1}(0)$ is principal means that

$T^{n}(x)=x \quad$ and $\quad n \geq 1 \quad \Rightarrow \quad \varphi_{n}(x) \equiv \varphi(x)+\varphi(T x)+\ldots+\varphi\left(T^{n-1} x\right) \neq 0$.

It implies that $\varphi$ is not of the form $\psi \circ T-\psi$. This condition is trivially satisfied when $\varphi$ is strictly positive (or strictly negative).

Corollary 3.3. Let $T: X \rightarrow X$ be a local homeomorphism and let $\varphi \in C(X ; \mathbf{R})$. Let $\alpha$ denote the action associated to the cocycle $c_{\varphi} \in Z^{1}(G, \mathbf{R})$. If $c_{\varphi}^{-1}(0)$ is principal, then every KMS state for $\alpha$ at inverse temperature $\beta$ is of the form $\omega_{\mu}$ for some quasi-invariant probability measure $\mu$ with Radon-Nikodym derivative $d r^{*} \mu / d s^{*} \mu=e^{-\beta c_{\varphi}}$.

Remark 3.4. In some cases, one can reach the same conclusion without any assumption on $\varphi$. For example, if the restriction of the KMS state to $C(X)$ induces a probability measure on $X$ with no atoms and if $T$ is a local homeomorphism which is positively expansive and exact, one also obtains that every KMS state is of the form $\omega_{\mu}$ (using the fact that periodic points of a given period are also isolated).

We are now ready to state the main result concerning the existence and uniqueness of KMS states for the action associated to the cocycle $c_{\varphi}$. A key step is to show that $P(T,-\beta \varphi)=0$ has a unique solution (see Corollary 4.3 of [R3] and following remarks).

Theorem 3.5. Let $T: X \rightarrow X$ be a local homeomorphism which is positively expansive and exact. Let $\varphi \in C(X ; \mathbf{R})$ and let $\alpha$ be the automorphism group associated to the cocycle $c_{\varphi} \in Z^{1}(G, \mathbf{R})$.

(i) There is a KMS state for $\alpha$ at inverse temperature $\beta \in \mathbf{R}$ if and only if $P(T,-\beta \varphi)=0$.

(ii) Let $\beta \in \mathbf{R}$ such that $P(T,-\beta \varphi)=0$. If $c_{\varphi}^{-1}(0)$ is principal and $\varphi$ satisfies the Bowen condition, then the KMS state at inverse temperature $\beta$ is unique.

(iii) Assume that $c_{\varphi}^{-1}(0)$ is not principal and that $\varphi$ satisfies the Walters condition. Then, for all $\beta \in \mathbf{R}, P(T,-\beta \varphi)>0$. In particular, $\alpha$ has no KMS state.

(iv) If $\varphi$ is strictly positive (resp. strictly negative), there is a unique $\beta$ such that $P(T,-\beta \varphi)=0$ and therefore a unique inverse temperature $\beta$ at which KMS states exist; moreover, $\beta>0$ (resp. $\beta<0$ ). Indeed we have

$$
\frac{h(T)}{\sup \varphi} \leq \beta \leq \frac{h(T)}{\inf \varphi} .
$$

If furthermore $\varphi$ satisfies the Bowen condition, the KMS state at inverse temperature $\beta$ is unique.

Proof. For (i), we note that if $\omega$ is a KMS state for $\alpha$ at inverse temperature $\beta$, the measure $\mu$ on $X$ given by its restriction to $C(X)$ is quasi-invariant with RadonNikodym derivative $e^{-\beta c_{\varphi}}$. This implies ([R3, Proposition 4.2]) that $\mathcal{L}_{-\beta \varphi}^{*} \mu=\mu$ and therefore, according to Proposition 2.5, that $P(T,-\beta \varphi)=0$. Conversely, if $P(T,-\beta \varphi)=0$, the same proposition gives the existence of a quasi-invariant probability measure $\mu$ with Radon-Nikodym derivative $e^{-\beta c_{\varphi}}$. Then, $\omega_{\mu}$ is a KMS state for $\alpha$ at inverse temperature $\beta$. For (ii), we note that the assumption that 
$c_{\varphi}^{-1}(0)$ is principal guarantees that every KMS state is of the form $\omega_{\mu}$, where $\mu$ is a Perron-Frobenius eigenvector $\mathcal{L}_{-\beta \varphi}^{*} \mu=\mu$ and the second assumption guarantees the uniqueness of this Perron-Frobenius eigenvector. For (iii), we assume that $c_{\varphi}^{-1}(0)$ is not principal and that $\varphi$ satisfies the Walters condition. According to (ii) of Theorem [2.8, there is $h \in C(X, \mathbf{R}), h>0$, such that $\mathcal{L}_{\varphi} h=\lambda h$, where $\log \lambda=P(T, \varphi)$. Since $c_{\varphi}^{-1}(0)$ is not principal, there exists $n \geq 1$ and $x \in X$ such that $T^{n} x=x$ and $\varphi_{n}(x)=0$. Then,

$$
\lambda^{n} h(x)=\mathcal{L}_{\varphi}^{n} h(x)=\sum_{T^{n} y=x} e^{\varphi_{n}(y)} h(y)>h(x) .
$$

Therefore $\lambda>1$ and $P(T, \varphi)>0$. Similarly $P(T,-\beta \varphi)>0$ for all $\beta \in \mathbf{R}$. According to (i), this implies that $\alpha$ has no KMS states. For (iv), assume that $\varphi$ is strictly positive. Then, the uniqueness of $\beta$ such that $P(T,-\beta \varphi)=0$ follows by the properties of pressure enumerated in [W2, Theorem 9.7]. We have $P(T, 0)=$ $h(T)>0$ (by Corollary 2.6) and $P(T,-\beta \varphi) \leq h(T)-\beta \inf \varphi<0$ for $\beta$ sufficiently large. The continuity of $\beta \mapsto P(T,-\beta \varphi)$ and the intermediate value theorem give the existence of $\beta>0$ such that $P(T,-\beta \varphi)=0$. The convexity of this function implies the uniqueness of the solution. The case for strictly negative $\varphi$ is handled similarly. The inequality follows immediately from [W2, Theorem 9.7(ii)]. Since the strict positivity (or negativity) of $\varphi$ implies that $c_{\varphi}^{-1}(0)$ is principal, we can apply (ii) to obtain the uniqueness when $\varphi$ also satisfies the Bowen condition.

Remark 3.6. If $\varphi$ is chosen to be the constant function 0 , then $\alpha$ is the identity automorphism group and (iii) says that $C^{*}(X, T)$ has no tracial state. As noted above it is well known that this $\mathrm{C}^{*}$-algebra is simple and purely infinite (see $\mathrm{An}$ ). If $\varphi$ is chosen to be the constant function 1, then $\beta=h(T)$, the topological entropy (see 3.5(iv)). Since $\varphi$ trivially satisfies the Bowen condition, there is a unique KMS state; this restricts to a trace on $C^{*}(R)$. The associated action $\alpha$ is the usual gauge action extended to $\mathbf{R}$. This generalizes results of Olesen and Pedersen (see [OP]).

KMS states may fail to exist if $\varphi$ is not strictly positive (or negative), as the next example shows. But this is not a necessary condition (see Example 3.10).

Example 3.7. Let $X$ and $T$ be as in Example 2.2 above; recall that $C^{*}(G) \cong \mathcal{O}_{n}$ is generated by $n$ isometries $S_{1}, \ldots, S_{n}$. Given real numbers $\lambda_{1}, \ldots, \lambda_{n}$ there is a (unique) one-parameter automorphism group $\alpha: \mathbf{R} \rightarrow \operatorname{Aut}\left(\mathcal{O}_{n}\right)$ such that $\alpha_{t}\left(S_{j}\right)=$ $e^{i t \lambda_{j}} S_{j}$ for $j=1,2, \ldots, n$. By [Ev, Prop. 2.2] there is a KMS state for $\alpha$ at inverse temperature $\beta$ iff

$$
1=\sum_{j=1}^{n} e^{-\beta \lambda_{j}}
$$

in this case the KMS state is unique. This condition holds for some $\beta$ exactly when $\lambda_{1}, \ldots, \lambda_{n}$ all have the same sign (i.e. all are positive or all are negative). Let us deduce these results from Theorem 3.5. We define $\varphi: X \rightarrow \mathbf{R}$ by $\varphi(x)=\lambda_{x_{0}}$. Then $\varphi$ is Hölder continuous and $\alpha$ is the one-parameter automorphism group associated to the cocycle $c_{\varphi}$. Note that we have

$$
\mathcal{L}_{-\beta \varphi} 1_{X}=\left(\sum_{j=1}^{n} e^{-\beta \lambda_{j}}\right) 1_{X} .
$$


Therefore, according to Proposition 2.5. $\sum_{j=1}^{n} e^{-\beta \lambda_{j}}$ is the exponential of the pressure $P(T,-\beta \varphi)$, and Evans' equation is equivalent to $P(T,-\beta \varphi)=0$. Thus, for a function $\varphi$ of that form, a KMS state exists if and only if $\varphi$ is strictly positive or strictly negative. Theorem 3.5 applies to give uniqueness. Note that there exist $\varphi \in C(X ; \mathbf{R})$ of the above form which do not have a constant sign but such that $c_{\varphi}^{-1}(0)$ is principal. This shows that the condition that $c_{\varphi}^{-1}(0)$ is principal is not sufficient for the existence of KMS states.

Example 3.8. Evans' example has been generalized to Cuntz-Krieger algebras by J. Zacharias in [Z]. Let $A$ be an irreducible $n \times n$ zero-one matrix $A=$ $(A(i, j))_{1 \leq i, j \leq n}$ and let $X_{A}$ and $T_{A}$ be as in Example 2.3. There are $n$ partial isometries $S_{1}, \ldots, S_{n}$ generating $C^{*}(G) \cong \mathcal{O}_{A}$, and one can define $\alpha: \mathbf{R} \rightarrow \operatorname{Aut}\left(\mathcal{O}_{A}\right)$ just as above. Zacharias has shown in [Z, Prop. 4.3] that there is a KMS state for $\alpha$ if and only all the $\lambda_{i}$ have the same sign, and in this case the KMS state is unique. The inverse temperature $\beta$ satisfies the condition that the spectral radius of $D_{\beta} A$ is 1 , where $D_{\beta}$ is the diagonal matrix with entries $e^{-\beta \lambda_{i}}$. This can be deduced from Theorem [3.5 just as above. We define $\varphi$ as previously. We find that the pressure $P(T,-\beta \varphi)$ is the logarithm of the the spectral radius of $D_{\beta} A$ and that it can take the value 0 if and only if all the $\lambda_{i}$ have the same sign. Then, we can apply the part (iv) of the theorem.

Remark 3.9. KMS states with respect to gauge automorphism groups on CuntzKrieger algebras have also been studied by D. Kerr and C. Pinzari in KP, Section 7] and by R. Exel in Ex2. Given a primitive $n \times n$ matrix $A$ with zero-one elements, we define $X=X_{A}$ and $T=T_{A}$ as in Example 2.3 and $\mathcal{O}_{A} \cong C^{*}\left(G\left(X_{A}, T_{A}\right)\right)$. The automorphism group $\alpha^{b, a}$, where $b \in \mathbf{R}$ and $a \in C(X, \mathbf{R})$, considered in $[\mathrm{KP}]$, is our automorphism group $\alpha$ for $\varphi=b-a$. They consider the KMS problem for $\alpha^{b, a}$ at inverse temperature 1 . Our necessary and sufficient condition for existence $P\left(T_{A},-\varphi\right)=0$ becomes $b=P\left(T_{A}, a\right)$. In order to obtain the uniqueness of the KMS state, they assume that the variation of $a$ is strictly less than the entropy $P\left(T_{A}, 0\right)$. Since this condition implies the strict positivity of $\varphi=b-a$, their results are covered by Theorem 3.5. The existence and uniqueness of KMS states in the case where $(X, T)=\left(X_{A}, T_{A}\right)$ and $\varphi$ is Hölder continuous and strictly positive also appears in [Ex2, Theorem 4.4].

Example 3.10. Here is an example where the KMS state is unique but the potential $\varphi$ does not have a constant sign. Let $X$ and $T$ be as in Example 2.2 with $n=2$. Let $\varphi \in C(X ; \mathbf{R})$ be defined by

$$
\varphi(x)= \begin{cases}\log (4 / 3) & \text { if } x_{0}=0 \text { and } x_{1}=1 \\ -\log 3 & \text { otherwise }\end{cases}
$$

Then we claim that $\alpha$ as defined above has a unique KMS state which occurs at inverse temperature $\beta=-1$. We write $(X, T)$ as the shift associated to the stationary Bratteli diagram given by the matrix

$$
\left(\begin{array}{ll}
1 & 1 \\
1 & 1
\end{array}\right)
$$

Then $c_{\varphi}$ is the stationary quasi-product cocycle given by the matrix

$$
\left(\begin{array}{cc}
-\log 3 & \log (4 / 3) \\
-\log 3 & -\log 3
\end{array}\right)
$$


According to Proposition 2.5] and [ $\mathrm{R} 3$, Section 3.2], the pressure $P(T,-\beta \varphi)$ is the logarithm of the spectral radius of the matrix

$$
B_{\beta}=3^{\beta}\left(\begin{array}{cc}
1 & 4^{-\beta} \\
1 & 1
\end{array}\right) \text {. }
$$

It is a strictly increasing function from $-\infty$ to $+\infty$, and it takes the value 0 at $\beta=-1$. In this example, $c_{\varphi}^{-1}(0)$ is principal, $\varphi$ does not have a constant sign and $\alpha$ has a unique KMS state.

Remark 3.11. Let $T: X \rightarrow X$ be a local homeomorphism, let $\varphi \in C(X ; \mathbf{R})$ and let $\alpha$ denote the action associated to the cocycle $c_{\varphi} \in Z^{1}(G, \mathbf{R})$. Then the subalgebra $C^{*}\left(R_{n}\right)$ is invariant under $\alpha$ and the restriction of $\alpha$ to $C^{*}\left(R_{n}\right)$ is inner (this is because the restriction of $c_{\varphi}$ to $R_{n}$ is a coboundary). It follows then that the restriction of $\alpha$ to $C^{*}(R)$ is approximately inner. It follows by $\mathrm{P}$, Proposition 8.12.9] that $C^{*}(R)$ has a ground state. Moreover, one may use the methods of [R5] to show that KMS states for the restriction of $\alpha$ to $C^{*}(R)$ exist at every inverse temperature $\beta \in \mathbf{R}$. This generalizes Propositions III.1.5 and III.2.9 of [R1].

\section{THE CROSSED PRODUCT}

We collect here some facts concerning the crossed product $C^{*}(G) \rtimes_{\alpha} \mathbf{R}$. If $T: X \rightarrow X$ is a local homeomorphism and $\varphi \in C(X ; \mathbf{R})$, then $\tilde{T}: X \times \mathbf{R} \rightarrow X \times \mathbf{R}$ defined by $\tilde{T}(x, t)=(x, t+\varphi(x))$ is a local homeomorphism. Moreover, $G(X \times \mathbf{R}, \tilde{T})$ may be identified with the skew product groupoid $G\left(c_{\varphi}\right)$, and so $C^{*}(G) \rtimes_{\alpha} \mathbf{R} \cong$ $C^{*}(G(X \times \mathbf{R}, \tilde{T}))$ (see DKM, Example 4]).

Note that $G$ is minimal but $G(X \times \mathbf{R}, \tilde{T})$ may well not be. We consider a necessary and sufficient condition for the minimality of $G(X \times \mathbf{R}, \tilde{T})$ : For every $x \in X$, open set $U \ni x, t \in \mathbf{R}$ and $\epsilon>0$, there exist $y \in U, m, n \in \mathbf{N}$ such that $T^{m} x=T^{n} y$ and $\left|c_{\varphi}(x, m-n, y)-t\right|<\epsilon$. This condition is equivalent to the requirement that $R_{\infty}^{x}\left(c_{\varphi}\right)=\mathbf{R}$ for all $x \in X$ (see [R1, Definition I.4.3]). Hence, $G(X \times \mathbf{R}, \tilde{T})$ is minimal and thus $C^{*}(G) \rtimes_{\alpha} \mathbf{R}$ is simple if and only if this condition is satisfied (see [R1, Proposition I.4.14]). This condition may be difficult to check, but see the examples below. Simplicity of the crossed product also has interesting consequences. The following proposition is a restatement of some earlier results appropriate to the current context:

Proposition 4.1. Let $X$ be a compact metric space and let $T: X \rightarrow X$ be a local homeomorphism which is positively expansive and exact. Let $\varphi \in C(X ; \mathbf{R})$ and let $\alpha$ be the one-parameter automorphism group associated to the cocycle $c_{\varphi} \in Z^{1}(G, \mathbf{R})$. Then

(i) If $C^{*}(G) \rtimes_{\alpha} \mathbf{R}$ is simple, then it is stable and either infinite or projectionless.

(ii) If $\varphi$ is strictly positive (or strictly negative) there is a faithful lower semicontinuous trace $\tau$ on the crossed product $C^{*}(G) \rtimes_{\alpha} \mathbf{R}$ which satisfies the scaling property with respect to the dual automorphism, i.e. $\tau \circ \hat{\alpha}_{s}=e^{-\beta s} \tau$ for all $s \in \hat{\mathbf{R}}$. It follows that the crossed product is stably projectionless.

Proof. Assertion (i) follows from [KK2, Props. 3, 4] and the fact that $C^{*}(G)$ is a unital simple purely infinite $\mathrm{C}^{*}$-algebra (see $\mathrm{An}$ ). Assertion (ii) follows from the existence of KMS states (see Theorem [3.5 v), [KK1, Theorem 3.2] and [KK1, Corollary 3.4]. 
Remark 4.2. In the setting of part (ii) of the above proposition it may be worthwhile to find an explicit formula for the trace on the crossed product constructed from a measure resulting from Proposition 2.5. Note that by arguing as in the proof of Theorem 3.5 there is a $\beta>0$ such that $P(T,-\beta \varphi)=0$. Let $\mu$ be a measure on $X$ such that

$$
\mathcal{L}_{-\beta \varphi}^{*} \mu=\mu
$$

(see the proof of Theorem $[3.5$ ); note that $\mu$ has full support. The measure $\tilde{\mu}=$ $\mu \times e^{-\beta t} d t$ on $X \times \mathbf{R}$ is invariant under the groupoid $G(X \times \mathbf{R}, \tilde{T})$ and therefore defines a faithful lower semicontinuous trace $\tau_{\omega}$ on the crossed product: it is easy to check that it satisfies the scaling property (see [KK1, Theorem 3.2])

$$
\tau_{\omega} \circ \hat{\alpha}_{s}=e^{-\beta s} \tau_{\omega} .
$$

It follows that $C^{*}(G) \rtimes_{\alpha} \mathbf{R}$ is stably projectionless.

Examples 4.3. In the case of Example 3.7 with $\lambda_{i}$ all of the same sign, Kishimoto has shown that $C^{*}(G) \rtimes_{\alpha} \mathbf{R}$ is simple if and only if the subgroup generated by the $\lambda_{i}$ is dense in $\mathbf{R}$ (see $\overline{K s}$ ); in this case it is also stably projectionless (see KK1, Th. 4.1]). In the case of Example 3.8 with $A$ primitive and $\lambda_{i}$ all of the same sign, Zacharias has shown that $C^{*}(G) \rtimes_{\alpha} \mathbf{R}$ is simple and stably projectionless if the $\lambda_{i}$ are linearly independent over Z (see [Z, Prop. 4.6]).

\section{REFERENCES}

[An] C. Anantharaman-Delaroche, Purely infinite $C^{*}$-algebras arising from dynamical systems, Bull. Soc. Math. France 125 (1997) 199-225. MR1478030 (99i:46051)

[AH] N. Aoki and K. Hiraide, Topological theory of dynamical systems. Recent advances, North-Holland Mathematical Library 52, North-Holland, Amsterdam, London, New York, Tokyo, 1994. MR1289410 (95m:58095)

[BEK] O. Bratteli, G. A. Elliott, and A. Kishimoto, The temperature state space of a $C^{*}$ dynamical system, I, Yokohama Math. J. 28 (1980) 125-167. MR.0623757 (82i:46101)

[BR] O. Bratteli and D. Robinson, Operator algebras and quantum statistical mechanics. 2: Equilibrium states. Models in quantum statistical mechanics, Second Ed., Texts and Monographs in Physics, Springer-Verlag, Berlin, 1997. MR 1441540 (98e:82004)

[Co] A. Connes, An analogue of the Thom isomorphism for crossed products of a $C^{*}$-algebra by an action of R, Adv. Math. 39 (1981) 31-55. MR0605351 (82j:46084)

[Cu] J. Cuntz, Simple $C^{*}$-algebras generated by isometries, Commun. Math. Phys. 57 (1977) 173-185. MR0467330(57:7189)

[CK] J. Cuntz and W. Krieger, A class of $C^{*}$-algebras and topological Markov chains, Invent. Math. 56 (1980) 251-268. MR0561974 (82f:46073a)

[D] V. Deaconu, Groupoids associated with endomorphisms, Trans. Amer. Math. Soc. 347 (1995) 1779-1786. MR1233967 (95h:46104)

[DKM] V. Deaconu, A. Kumjian and P. Muhly, Cohomology of topological graphs and CuntzPimsner algebras, J. Operator Theory 46 (2001) 99-122. MR1870406 (2003a:46093)

[EFW] M. Enomoto, M. Fujii, and Y. Watatani: KMS states for gauge action on $O_{A}$, Math. Japon. 29 (1984) 607-619. MR0759450 (86b:46110)

[Ev] D. E. Evans, On $\mathcal{O}_{n}$, Publ. R. I. M. S., Kyoto Univ. 16 (1980) 915-927. MR0602475 (82g:46099)

[Ex1] R. Exel, Crossed products by finite index endomorphisms and KMS states, J. Funct. Analysis 199 (2003), no. 1, 153-188. MR.1966826 (2004b:46094)

[Ex2] R. Exel, KMS states for generalized gauge actions on Cuntz-Krieger algebras (An application of the Ruelle-Perron-Frobenius Theorem), Bol. Soc. Brasil. Mat. (N.S.) 35 (2004), no. 1, 1-12. MR2057042 (2005c:46096)

[Ex3] R. Exel and A. Lopes, $C^{*}$-Algebras, Approximately Proper Equivalence Relations, and Thermodynamic Formalism, Ergodic Theory Dynam. Systems 24 (2004), no. 4, 10511082. MR2085390 
[FJ] A. Fan and Y. Jiang, On Ruelle-Perron-Frobenius Operators. I. Ruelle Theorem, Commun. Math. Phys. 223 (2001) 125-141. MR.1860762 (2002i:37029a)

[HHW] R. Haag, N. M. Hugenholtz and M. Winnink, On the equilibrium states in quantum statistical mechanics, Comm. Math. Phys. 5 (1967) 215-236. MR0219283 (36:2366)

[KP] D. Kerr and C. Pinzari, Noncommutative pressure and the variational principle in CuntzKrieger-type $C^{*}$-algebras, J. Funct. Anal. 188 (2002) 156-215. MR1878635 (2003f:46110)

[KK1] A. Kumjian and A. Kishimoto, Simple stably projectionless $C^{*}$-algebras arising as crossed products, Can. J. Math. 48 (1996) 980-996. MR1414067 (98b:46072)

[KK2] A. Kishimoto and A. Kumjian, Crossed products of Cuntz algebras by quasi-free automorphisms, in "Operator algebras and their applications" (P. Fillmore and J. Mingo, Eds.), 173-192, Fields Institute Communications 13, Amer. Math. Soc., Providence, 1997. MR 1424962 (98h:46076)

[Ks] A. Kishimoto, Simple crossed products by locally compact abelian groups, Yokohama Math. J. 28 (1980) 69-85. MR0623751 (82g:46110)

[LN] M. Laca and S. Neshveyev, KMS states of quasi-free dynamics of Pimsner algebras, J. Funct. Anal. 211 (2004), no. 2, 457-482. MR2056837

[OP] D. Olesen and G. K. Pedersen, Some $C^{*}$-dynamical systems with a single KMS state, Math. Scand. 42 (1978) 111-118. MR0500150(80a:46041)

[P] G. K. Pedersen, $C^{*}$-algebras and their automorphism groups, Academic Press, London, 1979. MR0548006 (81e:46037)

[Pm] M. Pimsner, A class of $C^{*}$-algebras generalizing both Cuntz-Krieger algebras and crossed products by Z $\mathbf{Z}$, in "Free probability theory" (D. Voiculescu, Ed.), 189-212, Fields Institute Communications 12, Amer. Math. Soc., Providence, 1997. MR1426840 (97k:46069)

[Rd] W. Reddy, Expanding maps on compact metric spaces, Topology Appl. 13 (1982) 327334. MR0651514 (83d:54070)

[R1] J. Renault, A Groupoid approach to $C^{*}$-algebras, Lecture Notes in Mathematics 793, Springer-Verlag, Berlin, 1980. MR0584266 (82h:46075)

[R2] J. Renault, The ideal structure of groupoid crossed product $C^{*}$-algebras, J. Operator Theory 25 (1991) 3-36. MR1191252 (94g:46074)

[R3] J. Renault, Cuntz-like algebras, Proceedings of the 17th International Conference on Operator Theory (Timisoara 98), The Theta Foundation (2000). MR1770333 (2001g:46130)

[R4] J. Renault, AF-equivalence relations and their cocycles, to appear in the Proceedings of the OAMP Conference (Constanţa, 2001), the Theta Foundation.

[R5] J. Renault, The Radon-Nikodym problem for approximately proper equivalence relations, preprint.

[Ru] D. Ruelle, Thermodynamic formalism for maps satisfying positive expansiveness and specification, Nonlinearity 5 (1992) 1223-1236. MR.1192516 (94a:58115)

[T] J.-L. Tu, The Baum-Connes conjecture for amenable groupoids, in " $\mathrm{C}^{*}$-algebras, Münster, 1999" (J. Cuntz and S. Echterhoff, Eds.), Springer, Berlin, 2000. MR1798599 (2001j:46109)

[W1] P. Walters, Invariant measures and equilibrium states for some mappings which expand distances, Trans. Amer. Math. Soc. 236 (1978) 121-153. MR0466493 (57:6371)

[W2] P. Walters, An introduction to ergodic theory, Graduate Texts in Mathematics, 79, Springer-Verlag, New York-Berlin, 1982. MR0648108 (84e:28017)

[W3] P. Walters, Convergence of the Ruelle operator for a function satisfying Bowen's condition, Trans. Amer. Math. Soc. 353 (2001) 327-347. MR1783787 (2001g:37029)

[Z] J. Zacharias, Quasi-free automorphisms of Cuntz-Krieger-Pimsner algebras, in " $\mathrm{C}_{\text {*- }}$ algebras, Münster, 1999" (J. Cuntz and S. Echterhoff, Eds.), 262-272, Springer, Berlin, 2000. MR.1798602(2002a:46079)

Department of Mathematics, University of Nevada, Reno, Nevada 89557

E-mail address: alex@unr.edu

Départment de Mathématiques, Université D'Orléans, 45067 Orléans, France

E-mail address: renault@labomath.univ-orleans.fr 